\title{
Resonance Scattering of Electrons in $\mathrm{Ag}_{2} \mathrm{Te}$
}

\author{
F.F. Aliyev*, M.B. Jafarov, V.I. Eminova, G.Z. Asgerova and R.A. Hasanova \\ Institute of Physics, Azerbaijan National Academy of Sciences, H. Javid ave. 33, Baku, AZ-1143, Azerbaijan
}

(Received March 28, 2011)

\begin{abstract}
The temperature dependences of electric conductivity $\sigma$, the Hall coefficient $R$ of $p$ - $\mathrm{Ag}_{2} \mathrm{Te}$ in $4.2-200 \mathrm{~K}$ temperature interval for acceptor concentration $N_{\mathrm{a}} \leq 6 \times 10^{16} \mathrm{~cm}^{-3}$ were investigated. The minimum $\sigma(T)$ was observed for all samples in $\approx 50 \div 80 \mathrm{~K}$ temperature interval. It was observed that the depth of minimum is increased with $N_{\mathrm{a}}$ decrease. It was shown that the part resonance scattering of electrons in minimum of $\sigma(T)$ and maximum of $\left|\alpha_{n}(T)\right|$ region is $16-18 \%$.
\end{abstract}

PACS: 72.15.Jf, 72.25.Pa, 71.23.An, 71.55.-1, 85.60.Gz

\section{Introduction}

It is related in the paper [1] that resonance scattering of electrons on acceptor impurities does not take place in $n-\mathrm{Ag}_{2} \mathrm{Te}$ at the donor concentration of $N_{\mathrm{d}} \geq$ $6.2 \times 10^{16} \mathrm{~cm}^{-3}$. In the paper [2] electrical and thermoelectrical properties of $p-\mathrm{Ag}_{2} \mathrm{Te}$ in samples with the acceptor concentration of $N_{\mathrm{a}} \geq 6.25 \times 10^{16} \mathrm{~cm}^{-3}$ are investigated. At such concentrations of $N_{\mathrm{a}}$ indications of resonance scattering of electrons on acceptor impurities in $p-\mathrm{Ag}_{2} \mathrm{Te}$ make their appearance. The manifestations of the mentioned phenomenon in $\mathrm{Ag}_{2} \mathrm{Te}$ at concentrations of $N_{\mathrm{a}} \leq 6.25 \times 10^{16} \mathrm{~cm}^{-3}$ have not been studied as yet.

The present research is dedicated to the study of electrical and thermoelectrical properties of $p-\mathrm{Ag}_{2} \mathrm{Te}$ in samples with the acceptor concentration of $N_{\mathrm{a}} \leq 7.8 \times$ $10^{16} \mathrm{~cm}^{-3}$. In all the samples of $p-\mathrm{Ag}_{2} \mathrm{Te}$ two peculiarities are observed on temperature relationship of electric conduction $\sigma(T)$ in the $4.2 \div 200 \mathrm{~K}$ temperature range: a plateau at $T<40 \mathrm{~K}$ and a minimum at $T \approx 65 \mathrm{~K}$ (Fig. 1). As can be seen from Fig. 1, the depth of minimum $\sigma(T)$ increases with reduction in $N_{\mathrm{a}}$ : it is about $7 \%$ for $N_{\mathrm{a}} \approx 7.8 \times 10^{16} \mathrm{~cm}^{-3}$ and about $30 \%$ for $N_{\mathrm{a}}$ $\approx 4.2 \times 10^{16} \mathrm{~cm}^{-3}$.

\section{Results and discussion}

At $T<40 \mathrm{~K}$ the Hall coefficient $R$ does not depend on temperature, at $T \geq 40 \mathrm{~K}$ it diminishes and changes its conduction sign from $p$ to $n$ when $T$ is about $T \approx 65 \mathrm{~K}$, then it passes through maximum at $T \approx 80 \mathrm{~K}$ (Fig. 1). The temperature course of thermoelectric power $\alpha_{0}(T)$ fully conforms to the course of $R(T)$, i.e. before $T \approx 40 \mathrm{~K}$ $\alpha_{0}$ linearly increases with temperature, at $T \approx 65 \mathrm{~K} \alpha_{0}$ changes its sign, then a dependence $\alpha_{0}(T)$ goes through

\footnotetext{
* corresponding author; e-mail: farzali@physics.ab.az
}

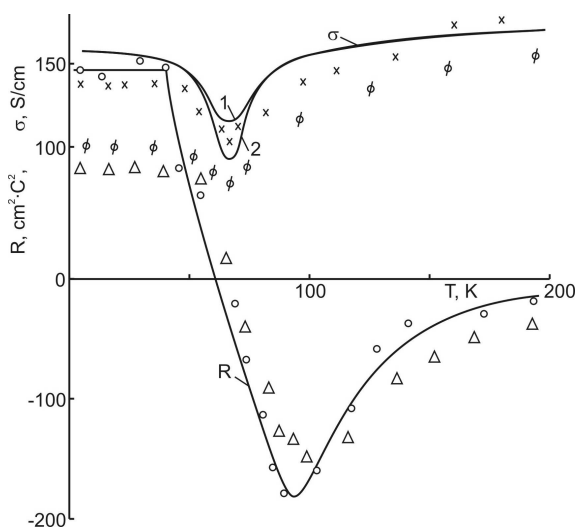

Fig. 1. Temperature relationships of electric conduction $\sigma$ and the Hall coefficient $R$ in $p-\mathrm{Ag}_{2} \mathrm{Te}$. Experimental points $(\phi, x-$ electric conduction, $o, \Delta-$ Hall coefficient for concentration of $p=7.8 \times 10^{16} \mathrm{~cm}^{-3}$, $p^{\prime}=4.2 \times 10^{16} \mathrm{~cm}^{-3}$, respectively) and solid lines are design points for concentration of $p^{\prime}=4.2 \times 10^{16} \mathrm{~cm}^{-3}$ : 1 - electron and hole scattering on ionic and acoustic vibrations, 2 - resonance scattering of electrons on acceptor impurities and hole scattering on acoustic vibrations of the lattice.

minimum at $T \approx 80 \mathrm{~K}$ (Fig. 2). The course of $\sigma(T), R(T)$ and $\alpha_{0}(T)$ at the concentration of $N_{\mathrm{a}} \approx 6.25 \times 10^{16} \mathrm{~cm}^{-3}$ is analyzed [2].

To reveal the cause of the depth of minimum $\sigma(T)$ dependence on $N_{\mathrm{a}}$ we shall perform an analysis of $\sigma$ and $\alpha_{0}$ within a two-zone model. As is known, $\sigma$ and $\alpha_{0}$ for two types of charge carriers are determined from the following formulae:

$$
\begin{aligned}
& \sigma=e\left(n U_{n}+p U_{p}\right)=\sigma_{n}+\sigma_{p}, \\
& \alpha_{0}=\frac{\alpha_{p} \sigma_{p}-\alpha_{n} \sigma_{n}}{\sigma_{p}+\sigma_{n}},
\end{aligned}
$$

where $U_{n}, U_{p}, n, p, \sigma_{n}, \sigma_{p}, \alpha_{n}$ and $\alpha_{p}$ are mobili- 


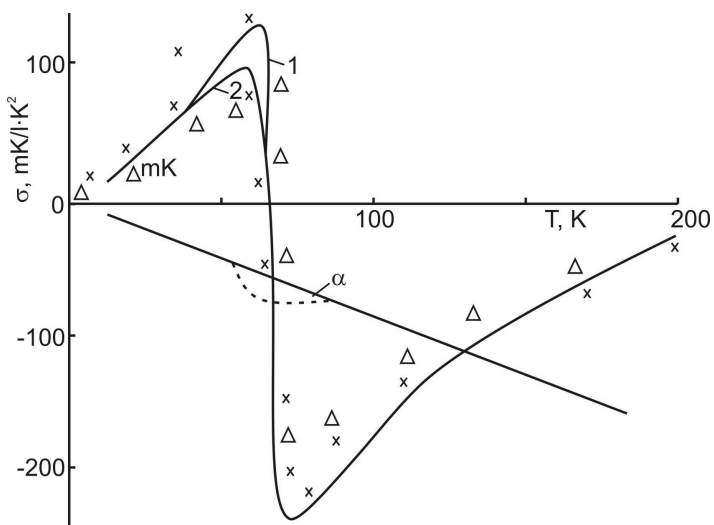

Fig. 2. Temperature relationship of thermoelectromotive force $\alpha_{0}(T)$ in $p$ - $\mathrm{Ag}_{2} \mathrm{Te}$. Experimental points (symbols are the same as in Fig. 1) and solid lines are design ones for concentration of $p^{\prime}=4.2 \times 10^{16} \mathrm{~cm}^{-3}$ : 1 electron and scattering on ionic and acoustic vibrations, 2 - resonance scattering of electrons on acceptor impurities and hole scattering on acoustic vibrations of the lattice.

ties and concentrations of electrons and holes, partial electroconductivities and thermoelectric power, respectively. Calculation of temperature relationship $U_{n}(T)$ and $U_{p}(T)$ in a sample of $N_{\mathrm{a}}=4.2 \times 10^{16} \mathrm{~cm}^{-3}$ in case of charge carrier scattering on ionic centres to the procedure in [2]; electron concentration was calculated from the formula

$$
n=\frac{n_{i}^{2}}{p_{0}+n_{i}},
$$

where $n_{i}$ - intrinsic concentration determined from $n_{i}=$ $4.9 \times 10^{15}\left(\frac{m_{n} m_{p}}{m_{0}}\right)^{3 / 4} T^{3 / 2} \exp \left(-\varepsilon_{\mathrm{g}} / 2 k_{0} T\right), p=p_{0}+n_{i}$ the total hole concentration. Here $p_{0}$ is determined from $R(T)$, where $R$ does not depend on temperature. By solving (2) electron concentration in the temperature interval under study is determined. It is found in paper [3] that valence zone is parabolic and then $p$ is determined according to [2]:

$$
p=\frac{\left(2 m_{p} k_{0} T\right)^{3 / 2}}{3 \pi^{2} \hbar^{3}} F_{3 / 2}\left(\mu_{p}^{*}\right) .
$$

It is found in the papers $[4,5]$ that dispersion law for electrons conforms to the Kane model, in this case electron concentration is determined in the following way:

$$
n=\frac{\left(2 m_{n} k_{0} T\right)^{3 / 2}}{3 \pi^{2} \hbar^{3}} I_{3 / 2}^{0}\left(\mu_{n}^{*}, \beta\right)
$$

where $m_{n}$ and $m_{p}$ are effective masses of electrons and holes, $F\left(\mu^{*}\right)$ and $I_{3 / 2}^{0}\left(\mu^{*}, \beta\right)$ one- and two-parameter Fermi integrals, $\mu^{*}=\frac{\mu}{k_{0} T}, \mu-$ chemical potential, $\beta=k_{0} T / \varepsilon_{\mathrm{g}}$ - parameter characterizing non-standard nature zone. The partial thermoelectric power for both cases are determined as

$$
\begin{aligned}
& \alpha_{p}=-\frac{k_{0}}{e}\left(\frac{F_{r+2}\left(\mu_{p}^{*}\right)}{F_{r+1}\left(\mu_{p}^{*}\right)}-\mu_{p}^{*}\right), \\
& \alpha_{n}=-\frac{k_{0}}{e}\left(\frac{I_{3 / 2,0}^{\prime}\left(\mu_{n}^{*}, \beta\right)}{I_{3 / 2,0}^{0}\left(\mu_{n}^{*}, \beta\right)}-\mu_{n}^{*}\right) .
\end{aligned}
$$

$\mu_{p}^{*}$ and $\mu_{n}$ were determined in (3), (4), $\alpha_{p}$ and $\alpha_{n}$ were calculated on their basis from the formulae (5). The partials $\sigma_{n}, \sigma_{p}$ were determined from the given $n, p, U_{n}$ and $U_{p}$ afterwards $\sigma(T)$ and $\alpha_{0}(T)$ were calculated using (1) (Figs. 1, 2). It is seen in Figs. 1 and 2 that in the $50 \div 70 \mathrm{~K}$ range the design curves of $\sigma(T)$ and $\alpha_{0}(T)$ do not quantitatively agree with the experimental ones. These disagreements can be analyzed as follows: at first we analyzed $\sigma(T)$. As seen from Fig. 3, at $T \leq 40 \mathrm{~K}$ hole mobility $U_{p}$ depends on temperature quite little while electron mobility $U_{n}$ goes up with temperature in accordance with the law: $U_{n} \sim T^{1.5}$. Such behavior of the mobilities demonstrates that at low temperatures charge carriers scatter on ionized impurities $[1,2]$, above $T>40 \mathrm{~K}$ the scattering takes place on acoustic vibrations of lattice thanks to which $\sigma(T)$ decreases in our opinion. The depth of minimum $\sigma(T)$ which is observed during experiments may be related to an abrupt decrease in $U_{n}$ in the $50 \div 65 \mathrm{~K}$ temperature range. It can be expected that electrons do not only scatter on ionized impurities and acoustic phonon (electron gas is not degenerated in this range) but also additional scattering mechanism is present.

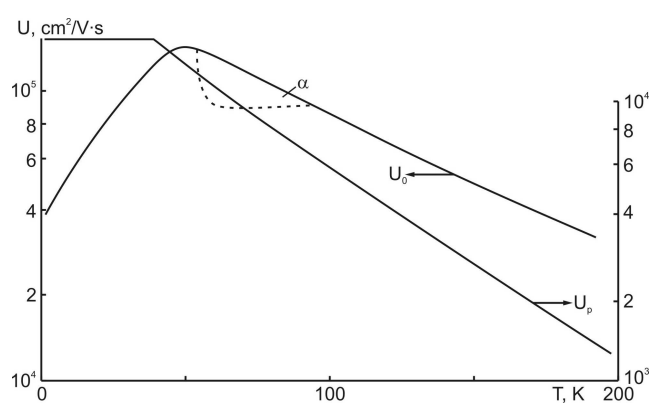

Fig. 3. Temperature relationship of electron $U_{n}(T)$ and hole $U_{p}(T)$ mobility in $p$ - $\mathrm{Ag}_{2}$ Te. Dotted lines are due to electron scattering on acceptor centres.

At low temperatures the average thermal energy of electron is much less than the donor level energy $E_{\mathrm{d}} \ll$ $k_{0} T\left(\varepsilon_{\mathrm{g}}=0.035 \mathrm{eV}, E_{\mathrm{d}}=0.002 \mathrm{eV}\right.$ and $E_{\mathrm{a}}=$ $0.004 \mathrm{eV}[6])$. With increase in temperature entrapment of electrons on acceptor states grows higher leading to a reduction in conduction electron number at $T<65 \mathrm{~K}$. It develops intensively provided that the Fermi level is located in a narrow area close to $E_{\mathrm{a}}$. When it is considered that the temperature relationship is $\varepsilon_{\mathrm{g}}=(0.035$ $\left.7 \times 10^{-5} \mathrm{~T} \mathrm{~K}^{-1}\right) \mathrm{eV}$, it can be expected that $\mu \rightarrow E_{\mathrm{a}}$. It allows to suppose that in this case electrons simultaneously scatter on acceptor centres (resonance scattering) as well. 
At resonance scattering $\sigma_{\text {rez }}$ takes the form [7]:

$$
\begin{gathered}
\sigma_{\mathrm{rez}}=\frac{\left(3 \pi^{2}\right)^{1 / 3} e^{2} p^{1 / 3}}{4 \pi \hbar N_{i}} \frac{m_{\mathrm{d}}^{*}}{m_{n}} \frac{\Gamma_{\mathrm{d}}}{\gamma} \\
\times\left[1+\tan ^{2}\left(\pi\left(k-\frac{1}{2}\right)\right)\right],
\end{gathered}
$$

where $m_{\mathrm{d}}^{*}$ - effective masses of state density, $p$ - hole concentration in valence zone, $\Gamma_{\mathrm{d}}$ - width of donor impurity band, $\gamma$ - broadening of resonance level at the expense of impurity and zone states hybridization. If the broadening of the level is completely due to non-stationary of impurity states then $\Gamma_{\mathrm{d}} / \gamma=1, k=$ $1 / 2+\left(N_{\mathrm{a}}-p\right) / 2 N_{i}$, where $N_{\mathrm{a}}$ and $N_{i}$ are concentrations of acceptors and impurity that had created a band the middle of which corresponds to the energy $E_{\mathrm{d}}$. If $\mu \rightarrow E_{\mathrm{a}}$ it can be assumed that $m_{\mathrm{d}} \rightarrow m^{*}$, where $m^{*}$ - effective mass of electrons at the Fermi level $\left(m^{*}=0.03 m_{0}\right.$ [8], $\left.m_{n}^{*}=0.02 m_{0}[2]\right)$. The width of $\Gamma_{\mathrm{d}}$ is determined from [7]:

$$
\rho_{i}(\varepsilon)=\frac{N_{i}}{\pi} \frac{\Gamma_{\mathrm{d}}}{\left(\varepsilon-\varepsilon_{i}\right)^{2}+\left(\Gamma_{\mathrm{d}} / 2\right)^{2}},
$$

where $\rho_{i}(\varepsilon)$ - density of impurity states, $N_{i}$ - concentration of impurity creating the band the middle of which corresponds to the energy $\varepsilon_{i}$. In the first approximation it can be taken that $\varepsilon_{i} \approx\left(E_{\mathrm{d}}-\mu\right) / 2$, at $T \ll E_{\mathrm{d}} / k_{0}, N_{i} \approx \frac{\left(2 m_{n} k_{0} T\right)^{3 / 2}}{3 \pi^{2} \hbar^{3}} \exp \left(-\left(\varepsilon_{\mathrm{d}}-\mu\right) / 2 k_{0} T\right)$.

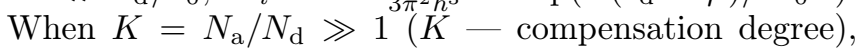
$\rho_{i}(\varepsilon)$ is determined [9]:

$$
\rho_{i}(\varepsilon)=\frac{N_{\mathrm{d}}}{\gamma_{0} \sqrt{\pi}} \exp \left(-\varepsilon^{2} / \gamma_{0}^{2}\right)
$$

where $\varepsilon \approx e^{2} / \chi r_{\mathrm{d}}$ - energy of the Coulomb interaction at the mid-distance between donors, $\chi$ - dielectric permittivity, $r_{\mathrm{d}}=\left(\frac{4 \pi}{3} N_{\mathrm{d}}\right)^{-1 / 3}$ - the average between donors

$$
\gamma_{0}=0.026 \varepsilon_{\mathrm{d}}\left(N_{\mathrm{A}}^{\prime} / N_{\mathrm{d}}^{\prime}\right)^{1 / 4},
$$

where $N_{\mathrm{A}}^{\prime}$ and $N_{\mathrm{d}}^{\prime}$ are concentrations of additional one-charge acceptors and donors not creating impurity levels.

From (7), (8) and (9) $\Gamma_{\mathrm{d}} \approx 0.2 \mathrm{meV}$ was obtained.

The broadening of band impurity level at the expense of impurity-zone transitions is determined from [10]:

$$
\gamma=\hbar / t
$$

where $t$ is the average life time of a carrier in impurity state relative to the transition to zone.

Proceeding from the detailed equilibrium principle one can equate frequencies of direct (zone-impurity) and reverse (impurity-zone) transitions [11]:

$$
\frac{\rho_{\mathrm{z}}}{\tau}=\frac{\rho_{i}}{t}
$$

where $\tau$ is the average life time of a carrier in zone state relative to the transition to impurity, $\rho_{\mathrm{z}}$ is density of zone states

$$
\rho_{\mathrm{z}}=\frac{\left(2 m_{n}\right)^{3 / 2} \mu^{1 / 2}}{2 \pi \hbar^{3}}
$$

and at low temperatures $\left(k_{0} T \ll \Gamma_{\mathrm{d}}\right) \tau$ depends on a scattering parameter

$$
r=\left.\frac{\partial \ln \tau}{\partial \ln \varepsilon}\right|_{\varepsilon=E_{\mathrm{d}}},
$$

where $r=\frac{2 \mu\left(\mu-\varepsilon_{i}\right)}{\left(\mu-\varepsilon_{i}\right)^{2}+\left(\Gamma_{\mathrm{d}} / 2\right)^{2}}$. A quantity $r$ has a minimum and a maximum at $\mu=\varepsilon_{i} \pm \Gamma_{\mathrm{d}} / 2$. A value of $t=6.6 \times 10^{-12} \mathrm{~s}$ was computed using values of $\rho_{\mathrm{z}}, \tau, \rho_{i}$, $\sigma_{\text {rez }}$ (Fig. 1) was computed with consideration for values of $\Gamma_{\mathrm{d}}, \gamma$ and $k$ in (6). It was seen from Fig. 1 that $\sigma_{\text {rez }}$ decreases less at the scattering on acoustic phonons.

Impurity states of $\mathrm{Ag}$ atoms $\left(E_{\mathrm{d}}=2 \mathrm{meV}\right)$ are located higher than the conduction band bottom; it could be expected from $T \approx 50 \mathrm{~K}$. However, at low temperatures $\left(T \ll E_{\mathrm{d}} / k_{0}\right)$ and donor concentrations of $N_{\mathrm{d}} \geq 6.2 \times 10^{16} \mathrm{~cm}^{-3}$ in $\mathrm{Ag}_{2}$ Te resonance scattering does not manifest itself. In this temperature range $\left(T \ll E_{\mathrm{d}} / k_{0}\right)$ electron scattering in the samples with $N_{\mathrm{d}} \geq 6.2 \times 10^{16} \mathrm{~cm}^{-3}$ (concentration of additional $\mathrm{Ag}$ atoms) is considerably weaker than in the samples with $N_{\mathrm{d}} \geq 4.2 \times 10^{16} \mathrm{~cm}^{-3}$ which can be explained by resonance scattering. With increase in Te atom content donor $N_{\mathrm{d}}$ concentration diminishes due to compensation. It leads to a decrease in an interval of donor smearing temperature and degeneration of electron gas reduces, too.

A strong dependence of impurity state density on energy brings about a sharp and non-monotonous energy dependence of relaxation time for resonance scattering which significantly affects the thermoelectric power $\alpha_{0}$ [9-12].

With concentration for non-parabolic of conduction band in $\mathrm{Ag}_{2} \mathrm{Te}$ a value of thermoelectromotive force is determined according to [7]:

$$
\alpha_{\mathrm{rez}}=\frac{k_{0}}{e} \frac{\pi^{2}}{3} \frac{k_{0} T}{\mu}\left(\gamma_{p}+\frac{3}{2} \frac{1+2 y}{1+y}-\frac{2 y}{1+2 y}\right),
$$

where $y=\mu / \varepsilon_{\mathrm{g}}$.

Calculation of $\alpha_{p}(T)$ from (13) in the $50 \div 70 \mathrm{~K}$ range is shown in Fig. 2.

It is seen from Figs. 1 and 2 that consideration for resonance scattering in $p$ - $\mathrm{Ag}_{2} \mathrm{Te}$ leads to an increase in design values of $\alpha_{n}$ and electrical resistance by about $16 \div$ $18 \%$. It follows from the ratio of the level $\gamma$ broadening at the expense of hybridization of impurity and zone states of $\gamma$ to the full width of $\Gamma_{\mathrm{d}}$ band, i.e. $\gamma / \Gamma_{\mathrm{d}} \approx 0.8$. As distinct from $n-\mathrm{Ag}_{2} \mathrm{Te}$, resonance scattering of electrons on acceptor impurities is detected in $p-\mathrm{Ag}_{2} \mathrm{Te}$ at donor concentrations of $N_{\mathrm{d}} \leq 4.2 \times 10^{14} \mathrm{~cm}^{-3}$.

The cause of an increase in resonance scattering contribution with decreasing $N_{\mathrm{a}}$ on $\sigma(T)$ may stem from the fact that a reduction in concentration of Te atoms (formation of $\mathrm{Ag}$ vacancy or addition of Te atoms to $\mathrm{Ag}_{2} \mathrm{Te}$ and their subsequent ionization always lead to $p$-type conduction [12]) results in a decrease in the width of prohibited zone $\varepsilon_{\mathrm{g}}$ and broadening of resonance level $\gamma$. There is a 


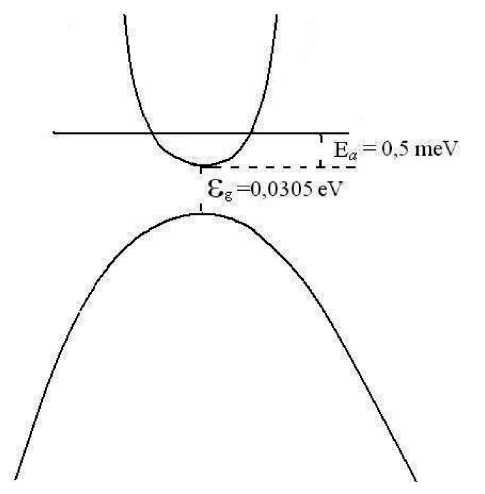

Fig. 4. Zone structure of $p-\mathrm{Ag}_{2}$ Te at $T=65 \mathrm{~K}$.

number of papers $[13,14]$, in which various values for $\varepsilon_{\mathrm{g}}$ in $\mathrm{Ag}_{2} \mathrm{Te}$ are presented. As the authors of [13] have obtained $\varepsilon_{\mathrm{g}} \leq 6 \mathrm{meV}$ in the region of intrinsic conduction, there applied reason to believe in a possibility of slot less state in $\mathrm{Ag}_{2} \mathrm{Te}$ at low temperatures. The authors of [14] have shown that $\mathrm{Ag}_{2} \mathrm{Te}$ becomes slot less in the $T>500 \mathrm{~K}$ temperature range. With consideration for negative value of temperature coefficient of the energy gap width $\varepsilon_{\mathrm{g}}$ it is evident that at lesser $\varepsilon_{\mathrm{g}}$ values acceptor level goes into conduction band. For this reason resonance scattering of electrons occurs and the depth of minimum $\sigma(T)$ increases.

Now we will find out the cause of $\sigma(T)$ increase and of the change of $\alpha_{0}(T)$ and $R(T) \operatorname{sign}$ at $T>65 \mathrm{~K}$ (Fig. 4). Calculations have demonstrated that at $T<$ $65 \mathrm{~K}$ the Fermi energy [2] is somewhat smaller than energy of acceptor basic state $\Delta E_{\mathrm{a}}$ and due to this electron concentration at the acceptor level $n_{\mathrm{a}}$ (where $\left.n_{\mathrm{a}}=N_{\mathrm{a}}\left[1+\frac{1}{2} \exp \left(-\frac{E_{\mathrm{a}}+\mu}{k_{0} T}\right)\right]^{-1}\right)$ remains constant. Width further growth in temperature (at $k_{0} T>E_{\mathrm{a}}$ ) the number of vacant places on acceptors reduces, i.e. generation of $n_{\mathrm{a}}$ starts and the process of electron excitation passes from valence zone to conduction band. The principal role in conduction within this temperature interval is played by concentration of $n_{\mathrm{a}}\left(n_{\mathrm{a}} \gg n_{i}\right)$ which rises exponentially at $T>65 \mathrm{~K}$ and that causes an increase in $\sigma$ and the change of $\alpha_{0}$ and $R$.

\section{Conclusion}

$p-\mathrm{Ag}_{2} \mathrm{Te}$ at the donor concentration of $N_{\mathrm{a}} \leq 6 \times$ $10^{16} \mathrm{~cm}^{-3}$ the electron resonance scattering on acceptor impurities has been revealed. It has been shown that at $T>65 \mathrm{~K}$ the electron concentration at the acceptor level, that at $T>65 \mathrm{~K}$ exponentially increases which leads to the growth of the electrical conductivity and the sign change of Hall coefficient and thermoelectric power, plays main role in conduction.

\section{References}

[1] F.F. Aliyev, E.M. Kerimova, S.A. Aliyev, Semiconductors 36, 869 (2002).

[2] F.F. Aliyev, M.B. Jafarov, Semiconductors 42, 1270 (2008).

[3] S.A. Aliyev, F.F. Aliyev, AS USSR News, Inorgan. Mater. 24, 341 (1988) (in Russian).

[4] S.A. Aliyev, U.H. Sugunov, M.I. Aliyev, Fiz. Tekn. Poluprovodn. 7, 2024 (1973) (in Russian).

[5] F.F. Aliyev, Semiconductors 37, 1057 (2003).

[6] V.I. Kaydanov, S.A. Nemov, Yu.I. Ravich, Fiz. Tekn. Poluprovodn. 26, 201 (1992) (in Russian).

[7] S.A. Aliyev, F.F. Aliyev, AS USSR News, Inorgan. Mater. 21, 1869 (1985).

[8] B.I. Shklovsky, A.L. Efros, Electronic Properties of Doped Semiconductors Nauka, Moskva 1979, p. 416.

[9] A.İ. Veis, S.A. Nemov, Fiz. Tekn. Poluprovodn. 15, 1237 (1981) (in Russian).

[10] S.A. Nemov, Yu.I. Ravich, Fiz. Tekn. Poluprovodn. 22, 1370 (1988) (in Russian).

[11] V.İ. Kaydonov, S.A. Nemov, Fiz. Tekn. Poluprovodn. 15, 542 (1981) (in Russian).

[12] H. Rau, J. Phys. Chem. Solids 28, 903 (1969); J. Phys. Chem. Solids 35, 1553 (1974).

[13] L.S. Koroleva, V.Yu. Martynov, P.P. Petrov, in: Proc. 2nd Conf. Material Science of Chalcogenide OxygenContaining Semiconductors, Ed. A.N. Georgobiani, Chernovtsy 1986, p. 47 (in Russian).

[14] S.A. Aliyev, Z.F. Agayev, R.I. Selimzade, Semiconductors 42, 1383 (2008). 